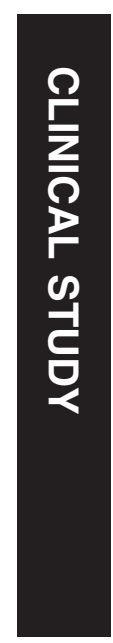

\title{
Intravitreal bevacizumab for the treatment of nonarteritic anterior ischemic optic neuropathy: a prospective trial
}

${ }^{1}$ Department of Ophthalmology and Vision Sciences, University of Toronto, Toronto, Ontario, Canada

${ }^{2}$ Department of Ophthalmology, Mount Sinai Hospital, Toronto, Ontario, Canada

Correspondence: EA Margolin, Department of Ophthalmology, Mount Sinai Hospital, Suite 409, 600 University Avenue, Toronto, Ontario, Canada M5G $1 \times 5$

Tel: + 1416586 4800;

Fax: + 4166195539

E-mail: edmargolin@ gmail.com

Received: 7 November 2012 Accepted: 14 December 2012

Published online: 1 February 2013

Presentations: Paper presented at the American Academy of Ophthalmology Annual Meeting, October 22-25, 2011, Orlando, FL.

\begin{abstract}
Purpose There is currently no accepted treatment for Nonarteritic Anterior Ischemic Optic Neuropathy (NAION). One new therapeutic approach involves decreasing optic nerve edema with intravitreal bevacizumab in order to resolve a proposed compartment syndrome. Methods In this non-randomized controlled clinical trial, $1.25 \mathrm{mg}$ intravitreal bevacizumab was compared with natural history. Patients were examined at baseline, 1, 3, and 6 months with a full neuro-ophthalmic exam, automated perimetry, and optic nerve optical coherence tomography (OCT) measurements. The primary outcome measure was change in mean deviation on Humphrey visual field testing. Secondary outcome measures were change in visual acuity and optic nerve OCT thickness. Incidence and type of complications were also recorded. Results Twenty-five patients were enrolled (17 treatment and 8 control). There was no significant effect of treatment on the primary outcome measure of mean deviation score $(P=0.4)$. There was similarly no effect of group assignment on the secondary outcome measures of change in mean Early Treatment Diabetic Retinopathy Study letters $(P=0.33)$ or nerve fiber layer thickness on OCT $(P=0.11)$. In the bevacizumab group, there was one case of a corneal abrasion and two cases of recurrent NAION. No other complications were noted.

Conclusions We found no difference between bevacizumab and natural history for change in visual field, visual acuity, or optic nerve OCT thickness. Based on the current
\end{abstract}

DB Rootman', HS Gill'1 and EA Margolin ${ }^{1,2}$

evidence we would not recommend the use of intravitreal bevacizumab to treat patients with the new onset of NAION.

Eye (2013) 27, 538-544; doi:10.1038/eye.2012.296; published online 1 February 2013

Keywords: optic nerve diseases; optic neuropathy, ischemic; eye diseases; vascular diseases; bevacizumab; angiogenesis inhibitors

\section{Introduction}

Currently, there is no accepted treatment for visual loss related to Nonarteritic Anterior Ischemic Optic Neuropathy (NAION). Although a wide range of medical and surgical approaches have been employed for this condition, most have demonstrated little benefit and some significant risk. ${ }^{1-6}$ However, one promising strategy involves the use of anti-inflammatory therapy in the acute stages of NAION. The theory behind this approach is that rapidly reducing swelling at the optic nerve head will reduce pressure in the crowded optic nerve head, thus opening up microvasculature and reducing post-ischemic nerve damage. ${ }^{7,8}$

Studies involving systemic ${ }^{9}$ steroids have demonstrated a $40 \%$ greater proportion of individuals improving $>0.30 \log$ MAR units. This study was, however, retrospective and only included individuals with presenting vision less that 20/70. Additionally, local steroid application is a more appealing strategy in that many of the systemic side effects can be avoided.

Studies involving intravitreal steroid injection ${ }^{10-13}$ have demonstrated improvement in visual acuity over $80 \%$ of reported cases. 
However, the efficacy of this strategy is ill defined, as the reported experience comes only from a small case series with no control group. ${ }^{10-12}$ In addition, although avoiding systemic toxicity, intravitreal steroids carry a significant risk of ocular complications such as glaucoma and cataract. ${ }^{14}$

Bevacizumab is a local therapy that has potent antiinflammatory properties, and has been used extensively to treat edema of the retina in a safe and efficacious manner. ${ }^{14,15}$ Recently, some authors have utilized this medication in acute NAION and found improvement in visual acuity in $>40 \%$ of patients. ${ }^{16,17}$ In almost $90 \%$ of these cases injections were performed within 14 days from symptom onset. However, these studies were small and uncontrolled, thus it is difficult to draw conclusions regarding this approach.

The purpose of the present investigation is to examine changes in visual field and visual acuity for patients affected by new onset NAION treated with intravitreal bevacizumab compared with the control group. We hypothesize that the intravitreal injection of bevacizumab within 14 days of symptom onset will improve visual outcome in individuals affected by NAION.

\section{Materials and methods}

\section{Protocol}

This study was approved by the Mount Sinai Hospital Research Ethics Board and a letter of no objection was obtained from Health Canada before the study commencement. All procedures were performed in accordance with the 1964 Declaration of Helsinki. The study was registered on clinicaltrials.gov (NCT00813059).

Consecutive patients presenting to one of the coauthors (EAM) with signs and symptoms consistent with NAION were examined and screened for study entry. Any clinical suspicion of an alternate cause for disk edema was investigated appropriately with blood work (including ESR and CRP) and imaging as required.

Inclusion criteria were: new onset NAION (under 15 days), age $>18$ and normal macula on clinical examination. Ocular exclusion criteria included: any other etiology to explain optic nerve disease, previous history of NAION in the same eye, previous history of other optic neuropathies, previous history of amblyopia in the affected eye, known glaucoma or clinical suspicion of glaucoma on presentation, ocular hypertension (IOP $>24 \mathrm{~mm} \mathrm{Hg}$ ), evidence of previous retinal vascular disease, and active uveitis. Non-ocular exclusion criteria included pregnancy, lactation, and inability to provide consent.
Eligible subjects were given an extensive explanation concerning their disease and the proposed study. They were then provided opportunity to consider study participation and consent was obtained.

All enrolled patients underwent baseline neuroophthalmological examination and investigations including measurements of best corrected visual acuity on Early Treatment Diabetic Retinopathy Study (ETDRS) chart, intraocular pressure measurements, pupillary, biomicroscopic, and dilated fundus examination, as well as formal field testing with Humphrey visual fields (24-2 SITA standard algorithm) and optical coherence tomography (OCT) of the optic nerves. OCT was performed with the Cirrus OCT device (Zeiss Meditec, Oberkochen, Germany).

After consent was obtained for study entry, patients were transferred to another coinvestigator for injection. Risks and benefits of injection were reviewed and patients were provided an opportunity to consider whether they would choose to be assigned to the treatment or control group. Participants consenting to treatment then underwent intravitreal injection of bevacizumab as described below. Individuals consenting for control group were followed without intervention.

\section{Intervention}

Bevacizumab (1.25 mg) was injected intravitreally by transcleral injection. Sterile tetracaine drops were instilled in the affected eye. The eye and surrounding periocular skin was then prepped with $5 \%$ betadine solution. Lidocaine $1 \%$ without epinephrine was injected subconjunctivally in the superotemporal quadrant adjacent to the anatomic limbus. A lid speculum was placed in the eye and the anesthetized area was cleaned with $5 \%$ betadine. A variable caliper was utilized to mark the pars plana, $4 \mathrm{~mm}$ from the limbus for phakic and $3.5 \mathrm{~mm}$ for pseudophakic patients. A 25-gauge needle was then introduced through this mark into the vitreous cavity and the bevacizumab was injected. The needle was retracted and the injection site covered with a sterile cotton tipped applicator. A drop of topical gatifloxacin was placed in the eye and the speculum removed. Patients were instructed to continue the gatifloxacin drops four times a day for 5 days.

\section{Study visits}

Patients were seen at baseline and underwent a comprehensive neuro-ophthalmological examination as noted above. Any clinical suspicion of macular edema was further assessed by macular OCT. A complete screening neurological exam was also performed. Any symptoms or signs suggesting an alternate etiology for 
optic nerve edema were explored with appropriate investigations including ESR, CRP, CBC, and MRI/MRA. ETDRS visual acuity measurement, Humphrey automated perimetry (24-2 algorithm), and optic nerve OCTs were also performed at baseline.

Subsequently, all patients were re-examined at 1,3, and 6 months. Follow-up examinations involved a comprehensive neuro-ophthalmological examination including ETDRS visual acuity and dilated fundoscopic examination. Humphrey visual fields and optic nerve OCTs were also repeated at each of these visits.

In addition to all study visits, those patients who underwent intervention were examined at 1 day and 1 week to monitor for complications related to the intraocular injection. A standardized checklist was developed to screen for possible complications. Specifically, the following adverse events were ruled out: elevated intraocular pressure, endophthalmitis, retinal tear, retinal detachment, vitreous hemorrhage, uveitis, and corneal abrasion. Any other complications encountered were also noted.

\section{Outcome measures}

The primary outcome measure was change in mean deviation on Humphrey visual field testing from baseline to 6 months. Patients were tested by experienced technicians who were masked to the study group assignment.

Secondary outcome measures were change in ETDRS visual acuity and mean optic nerve thickness on OCT from baseline to 6 months. Secondary outcome measures were similarly assessed by trained technicians masked to study group assignment. Optic nerve OCT was performed on a Zeiss Cirrus OCT device (Zeiss Meditec). Visual acuity was measured utilizing an electronic, wall-mounted ETDRS visual acuity display device.

\section{Analysis}

Using a last observation carried forward strategy, baseline, and final outcome measurements for the primary and secondary outcome variables were compared utilizing a repeated measures ANOVA design with one between subjects factor. The independent variable of group assignment was included for treatment effect. Any statistically significant baseline differences between groups were entered as covariates in the model.

Baseline characteristics were compared with Student's t-test, $\chi^{2}$, and Fisher's exact test as appropriate. All analyses were performed with IBM SPSS Statistics for Mac version 20.0 (SPSS Inc, an IBM Company, Somers, NY, USA).

\section{Results \\ Study visits}

Twenty $(80.0 \%)$ patients attended the 6-month follow-up examination. Three $(12.0 \%)$ patients attended at only 3 months. Four (16.0\%) cases were seen only at the 1-month time point. All data was analyzed with the last visit carried forward.

\section{Sample}

Thirty-two patients were screened for study entry. Based on clinical history, two patients were excluded due to previous history of NAION in the same eye. Four patients were excluded for onset greater than 15 days before examination. Of the 26 enrolled patients, one was excluded after study entry due to inability to complete any follow-up examinations despite multiple attempts to reschedule. This patient did not receive the study intervention. Of the 25 included cases, 17 elected to have the study intervention.

The mean (SD) age of patients was 60.8 (7.3) years and $68 \%(n=17)$ were male. A past medical history of noninsulin dependent diabetes was noted in $8.0 \%(n=2)$ and hypertension was found in $40.0 \%(n=10)$ of patients. One patient was being treated for hyperlipidemia.

Mean (SD) time from symptom onset to presentation was 8.4 (4.0) days; this was significantly $(P<0.05)$ shorter for the treatment group $(7.12 \pm 3.7)$ when compared with the control group (11.0 \pm 3.2 ) (Table 1). Median presenting visual acuity was equivalent to $20 / 30$, and the proportion (n) of patients presenting with better than 20/64 vision was $76 \%$ (19), with no significant differences between the groups $(P=0.345)$. There was no significant difference between outcome variables at baseline (Table 1).

\section{Primary and secondary outcomes}

For the primary outcome variable (Figure 1) of Humphrey visual field mean deviation score, there was

Table 1 Baseline characteristics

\begin{tabular}{lcc}
\hline & Control & Bevacizumab \\
\hline Patient characteristics & & \\
Non-insulin dependent diabetes & $0 \%(0)$ & $11.8 \%(2)$ \\
Hypertension & $14.3 \%(1)$ & $52.9 \%(9)$ \\
Smoking & $0 \%(0)$ & $5.9 \%(1)$ \\
Previous NAION & $0 \%(0)$ & $35.3 \%(6)$ \\
Age & $60.2(7.8)$ & $61.0(7.3)$ \\
Days from onset to examination & $11.0(3.3)$ & $7.1(3.8)^{*}$ \\
Baseline measurements & & \\
Mean deviation (SD) & & \\
Mean optic nerve OCT thickness (SD) & $250.6(114.6)$ & $226.7(80.6)$ \\
Mean number ETDRS letters (SD) & $58.5(45.4)$ & $84.1(32.9)$ \\
\hline
\end{tabular}

Abbreviations: ETDRS, Early Treatment Diabetic Retinopathy Study; NAION, nonarteritic anterior ischemic optic neuropathy; OCT, optical coherence tomography. ${ }^{*} P<0.05$. 


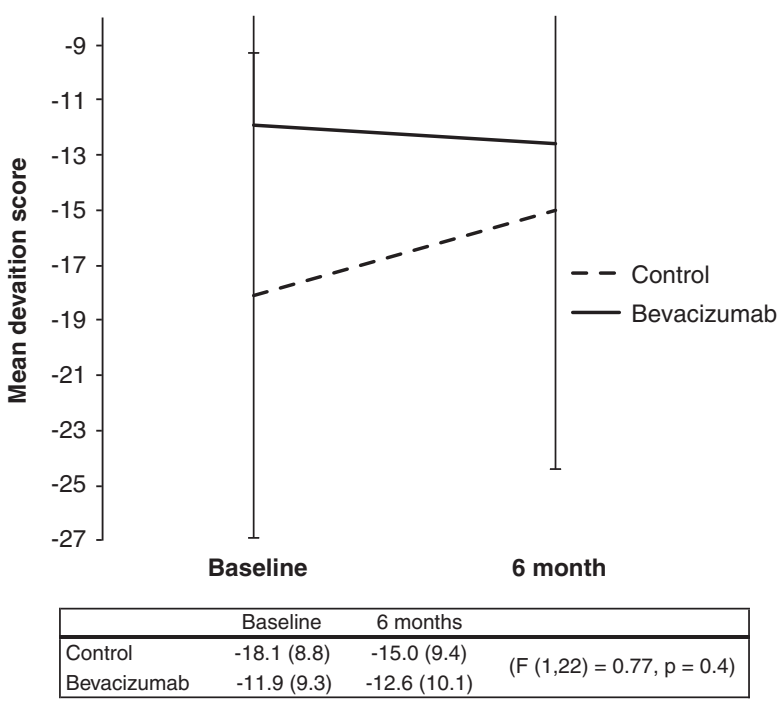

Figure 1 Mean Humphrey visual field mean deviation score at baseline and 6-month follow-up for control and bevacizumab arms. Error bars represent SD. Inset: Mean (SD) mean deviation score and results of repeated measures ANOVA.

no significant effect of time $(\mathrm{F}(1,22)=0.01, P=0.91)$. Additionally, the interaction effect of group assignment was not significant, indicating no differential effect of bevacizumab $(\mathrm{F}(1,22)=0.77, P=0.4)$.

For the secondary outcome measure of ETDRS visual acuity (Figure 2), no significant overall effect of time was noted $(\mathrm{F}(1,22)=0.35, P=0.57)$. Additionally, there was no effect of group assignment $(\mathrm{F}(1,22)=1.01, P=0.33)$.

There were three cases in which visual improvement was greater than 15 letters. Each of these cases had a presenting visual acuity worse than $20 / 64$. Three cases lost greater than 15 letters of vision. The differences in three lines of vision gain (Fisher's exact, $P=0.231$ ) and three lines of vision loss (Fisher's exact, $P=1.0$ ) were not significant between the treatment and control arms.

There was a decrease in mean OCT optic nerve thickness over time $(\mathrm{F}(1,11)=18.67, P<0.01)$; however, there was no difference in this change with treatment $(\mathrm{F}(1,11)=3.06, P=0.11)$ (Figure 3 ).

\section{Complications}

No cases of endophthalmitis were noted. No patient demonstrated an intraocular pressure $>20 \mathrm{~mm} \mathrm{Hg}$ at any study visit. One patient experienced a corneal abrasion as a result of the injection procedure that healed without sequelae. Two patients had a second NAION episode in the study eye during the study period. The first was 4 days after injection and the second was discovered outside of the study period at 14 months after treatment with bevacizumab. The difference in proportions of

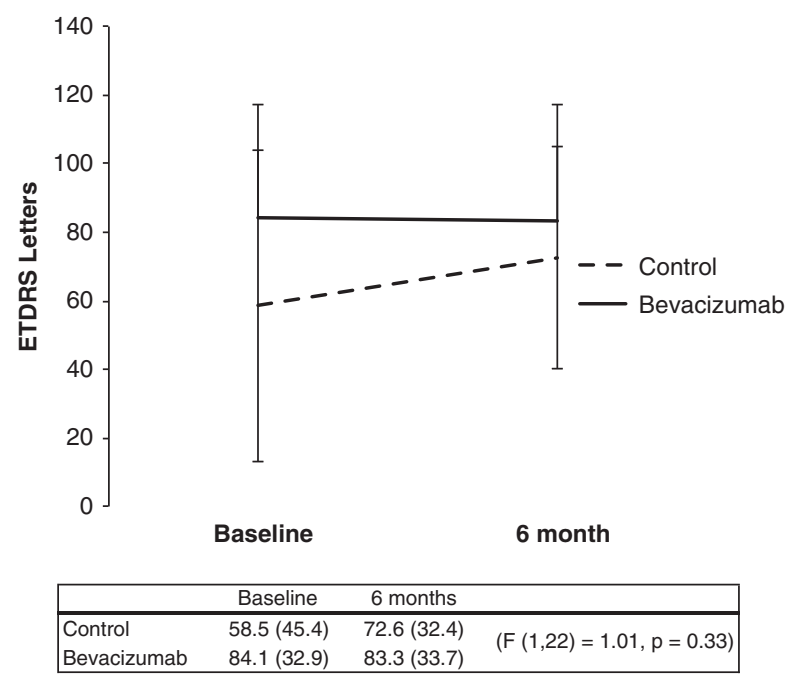

Figure 2 Mean number of ETDRS letters visual acuity at baseline and 6-month follow-up for control and bevacizumab arms. Error bars represent SD. Inset: Mean (SD) ETDRS letters and results of repeated measures ANOVA.

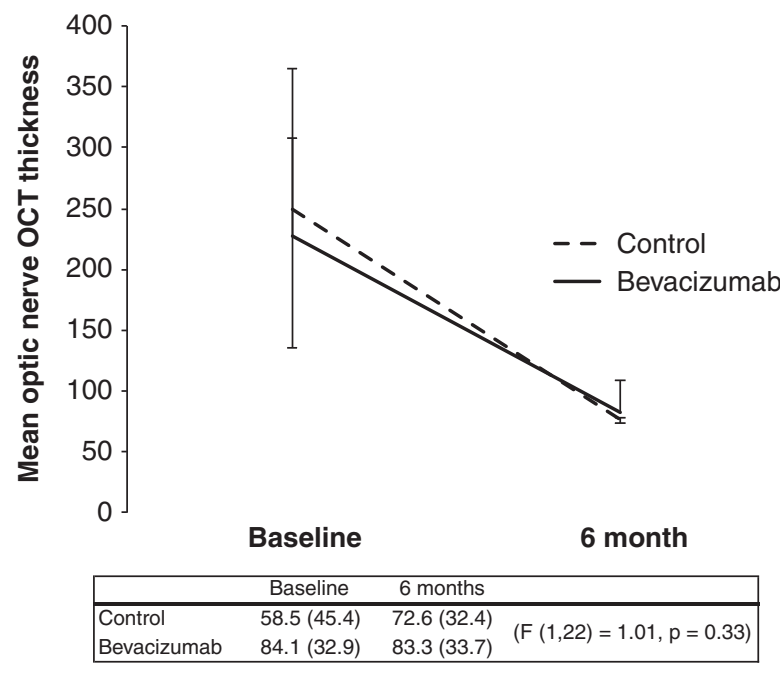

Figure 3 Mean optic nerve OCT mean thickness (in $\mu \mathrm{m}$ ) at baseline and 6-month follow-up for control and bevacizumab arms. Error bars represent SD. Inset: Mean (SD) optic nerve OCT thickness and results of repeated measures ANOVA.

recurrent NAION between the treatment and control groups was not statistically significant (Fisher's exact test, $P=1.0)$.

\section{Discussion}

In this prospective clinical study, our findings indicate that the injection of intravitreal bevacizumab did not significantly alter the course of new onset NAION relative to natural history. The primary outcome variable 
of Humphrey visual fields mean deviation score was not significantly affected by bevacizumab, nor were the two secondary outcome variables, visual acuity, or mean optic nerve OCT thickness. Importantly, there were two cases in which recurrent NAION occurred in the study eye.

We did not find a significant main effect of time on mean deviation or visual acuity. This is unlike previous reports of natural history that have demonstrated an improvement of greater than three lines of vision in $\sim 40 \%$ of patients. ${ }^{2,18}$ Our results demonstrate this finding in only $12 \%$ of patients overall. This disparity may be due to differences in patient population. In previous studies, ${ }^{2}$ patients with better than $20 / 64$ vision were excluded, and in our study these patients made up two-thirds of the sample. If we consider only those patients with visual acuity 20/64 or worse, then greater than three-line gain occurs in $50.0 \%$ of cases. In previous reports also studying a broad population, close to $10 \%$ of patients with vision 20/64 or better had a three-line improvement at 6 months. ${ }^{18}$ Additionally, studies with broader inclusion found little or no change overall in acuity or visual field. ${ }^{19,20}$ Our study tends to support these results in that individuals with vision worse than 20/64 at presentation may be more likely to experience greater than three-line improvement in visual acuity, and for samples including presenting vision better than 20/64 the overall improvement in vision and/or visual filed may be expected to be more tempered.

In prior studies that examined bevacizumab as a possible treatment option for NAION, Kelman et al ${ }^{17}$ presented at the North American NeuroOphthalmological Society 12 cases of patients treated with intravitreal bevacizumab within 14 days of symptom onset. Of these, five had a greater than threeline improvement in vision at 1 month. The discrepancy between Kelman's results and those from our study may be related to differences in the sample composition. Two-thirds of our sample had presenting acuity of $>20 / 64$, and may be less likely to achieve a three-line improvement in visual acuity than those presenting at lower visual levels, as noted above. ${ }^{18}$ The presenting acuity in their study was not reported. Additionally, with our longer follow-up, it is possible that some of the threeline improvements at 1 month may have settled to a more modest improvement at 6 months. Finally, we described six patients with loss of vision $>15$ letters, which may have skewed our overall results towards more modest improvement.

Our results are more inline with the uncontrolled retrospective study of five patients described by Prescott et $a l^{21}$ who found visual acuity improvement in one case presenting with 20/150 vision. Of the remainder who presented with normal acuity, three experienced some decline in vision. Additionally, only one patient experienced improvement in visual filed.

Interestingly, they had one patient who presented with normal visual acuity and progressed to 20/400 1 week after injection of bevacizumab. It is plausible that this patient may have experienced some progression of the optic nerve disease, extending the visual field defect through fixation. However, another possibility is that the patient experienced a sequential NAION in this eye. In our study we describe two such cases.

This finding of recurrent NAION after bevacizumab is concerning. Although sequential NAION in the fellow eye occurs in $15 \%$ of patients, ${ }^{22}$ recurrence in the same eye is rarely encountered, with prevalence estimates of $<6 \%{ }^{19,20,22-24}$ These recurrences are usually greater than 3 months after initial episode. ${ }^{23}$ The incidence of two $(11.7 \%)$ in our sample of treated eyes, one of which occurred within a few days of injection, raise suspicion that the injection itself may have had a role.

A postulated etiologic relationship between NAION and bevacizumab injection is supported by three additional published case reports. In the first, a patient with small cupless disks experienced an episode of NAION 2 weeks after injection of bevacizumab. These authors suggest that the cause could be an ischemic episode due to anti-VEGF activity or coincidence. ${ }^{25} \mathrm{~A}$ second study of a diabetic patient, reported an episode of NAION 3 weeks after bevacizumab injection for diabetic macular edema. They also cite microvascular changes secondary to VEGF blockade being causative for this association. ${ }^{26}$

A third report describes a woman who experienced NAION in one eye previously and was being treated for subfoveal choroidal neovascularization related to macular degeneration in the other eye. This patient experienced an episode of NAION in the bevacizumab treated eye 1 week after the second treatment cycle. These authors postulated that the recurrent NAION may be related to impaired vascular autoregulation attributable to VEGF blockade or acute IOP elevation. ${ }^{27}$ Concerning IOP elevation, the timing seems somewhat incongruous with this, as increases in IOP post-injection are typically transient, usually dissipating within 30 min, ${ }^{28,29}$ which is much earlier than the recurrent NAION episodes reported.

Although coincidence is always a possibility in this rarely occurring entity, our results in combination with these case reports suggest that the association between bevacizumab injection and NAION in susceptible eyes may not be merely coincidental. We would also postulate the mechanism to be related to microvascular changes secondary to VEGF blockade. ${ }^{26,27}$ Further research into this question is warranted. 
There are a number of important limitations of this study that should be noted. Initially, there was no randomization. This approach was chosen to improve patient autonomy in the informed consent process, considering the early experimental nature of this study. Additionally, there was a significant difference in time from onset of symptoms to examination between the two groups, with the injection group presenting earlier. This may have influenced the results, as some change in vision may have occurred in the control group before presentation. ${ }^{18,19}$ We account for this difference through multivariate statistics. In addition, the efficacy of bevacizumab on early asymptomatic NAION, an entity that has been reported, ${ }^{30}$ would be difficult to assess. Finally, as a relatively small study, there is insufficient power to control for beta error, making determinations of equivalence difficult to assert. As a corollary, our study was also not sufficiently powered for safety.

Though underpowered for beta error, this small non-randomized experimental study has not demonstrated any difference in visual field, visual acuity, or optic nerve OCT mean thickness between NAION patients treated with intravitreal bevacizumab vs those observed without intervention. There was a higher incidence of recurrent NAION in the treatment eye, and although this difference was not statistically significant, its occurrence raises concern about intravitreal bevacizumab injection in susceptible patients. Overall, we are unable to conclude that patients with new onset NAION treated with bevacizumab have better outcomes that those simply observed through 6 months of their disease.

\section{Summary}

What was known before

- No accepted treatment for NAION-anti-inflammatory therapy may improve outcome-some promising results with bevacizumab

- Anti-inflammatory therapy may improve outcome

- Some promising results with bevacizumab

What this study adds

- Bevacizumab does not appear to improve outcome over natural history in NAION

\section{Conflict of interest}

The authors declare no conflict of interest.

\section{Acknowledgements}

This project was funded by a grant from the Helen Keller Foundation for Research \& Education.DisclosureAll authors confirm that this is original work, no part of which has been previously published. All authors have full control of all primary data and agree to allow Graefe's Archive for Clinical and Experimental Ophthalmology to review our data if requested.

\section{References}

1 Botelho PJ, Johnson LN, Arnold AC. The effect of aspirin on the visual outcome of nonarteritic anterior ischemic optic neuropathy. Am J Ophthalmol 1996; 121: 450-451.

2 Dickersin K, Everett D, Feldon S, Hooper F, Kaufman D, Kelman $\mathrm{S}$ et al. Optic-nerve decompression surgery for nonarteritic anterior ischemic optic neuropathy (naion) is not effective and may be harmful. JAMA 1995; 273: 625-632.

3 Kaufman D, Dickersin K, Kelman S, Langenberg P, Newman N, Wilson PD. Ischemic optic neuropathy decompression trial: twenty-four-month update. Arch Ophthalmol 2000; 118: 793-798.

4 Modarres M, Sanjari MS, Falavarjani KG. Vitrectomy and release of presumed epipapillary vitreous traction for treatment of nonarteritic anterior ischemic optic neuropathy associated with partial posterior vitreous detachment. Ophthalmology 2007; 114: 340-344.

5 Soheilian M, Koochek A, Yazdani S, Peyman GA. Transvitreal optic neurotomy for nonarteritic anterior ischemic optic neuropathy. Retin-J Retin Vitr Dis 2003; 23: 692-697.

6 Wilhelm B, Ludtke H, Wilhelm H, Grp BS. Efficacy and tolerability of $0.2 \%$ brimonidine tartrate for the treatment of acute non-arteritic anterior ischemic optic neuropathy (NAION): a 3-month, double-masked, randomised, placebo-controlled trial. Graefes Arch Clin Exp Ophthalmol 2006; 244: 551-558.

7 Arnold AC. Pathogenesis of nonarteritic anterior ischemic optic neuropathy. J Neuro-Ophthal 2003; 23: 157-163.

8 Hayreh SS, Zimmerman MB. Optic disc edema in non-arteritic anterior ischemic optic neuropathy. Graefes Arch Clin Exp Ophthalmol 2007; 245: 1107-1121.

9 Hayreh SS, Zimmerman MB. Non-arteritic anterior ischemic optic neuropathy: role of systemic corticosteroid therapy. Graefes Arch Clin Exp Ophthalmol 2008; 246: 1029-1046.

10 Jonas JB, Spandau UH, Harder B, Sauder G. Intravitreal triamcinolone acetonide for treatment of acute nonarteritic anterior ischemic optic neuropathy. Graefes Arch Clin Exp Ophthalmol 2007; 245: 749-750.

11 Kaderli B, Avci R, Yucel A, Guler K, Gelisken O. Intravitreal triamcinolone improves recovery of visual acuity in nonarteritic anterior ischemic optic neuropathy. J Neuroophthalmol 2007; 27: 164-168.

12 Yaman A, Selver OB, Saatci AO, Soylev MF. Intravitreal triamcinolone acetonide injection for acute non-arteritic anterior ischaemic optic neuropathy. Clin Exp Optom 2008; 91: 561-564.

13 Sohn BJ, Chun BY, Kwon JY. The effect of an intravitreal triamcinolone acetonide injection for acute nonarteritic anterior ischemic optic neuropathy. Korean J Ophthalmol 2009; 23: 59-61.

14 Elman MJ, Aiello LP, Beck RW, Bressler NM, Bressler SB, Edwards AR et al. Randomized trial evaluating ranibizumab plus prompt or deferred laser or triamcinolone plus prompt laser for diabetic macular edema. Ophthalmology 2010; 117: 1064-1077.e35. 
$15 \mathrm{Wu}$ L, Arevalo JF, Berrocal MH, Maia M, Roca JA, Morales-Cantón V et al. Comparison of two doses of intravitreal bevacizumab as primary treatment for macular edema secondary to central retinal vein occlusion: results of the pan American collaborative retina study group at 24 months. Retin-J Retin Vitr Dis 2010; 30: 1002-1011.

16 Bennett JL, Thomas S, Olson JL, Mandava N. Treatment of nonarteritic anterior ischemic optic neuropathy with intravitreal bevacizumab. J Neuroophthalmol 2007; 27: 238-240.

17 Kelman S, Elman M, Mandava N, Bennett J. Intravitreal bevacizumab for nonarteritic anterior ischemic optic neuropathy: a pilot study. In: Proceeds from the Meeting of the North American Neuro-Ophthalmological Society Lake Tahoe, NV, 2009.

18 Hayreh SS, Zimmerman MB. Nonarteritic anterior ischemic optic neuropathy: natural history of visual outcome. Ophthalmology 2008; 115: 298-305.e2.

19 Boghen DR, Glaser JS. Ischemic optic neuropathy-clinical profile and natural-history. Brain 1975; 98: 689-708.

20 Sawle GV, James CB, Russell RWR. The natural-history of nonarteritic anterior ischemic optic neuropathy. J Neurol Neurosurg Psychiatry 1990; 53: 830-833.

21 Prescott CR, Sklar CA, Lesser RL, Adelman RA. Is intravitreal bevacizumab an effective treatment option for nonarteritic anterior ischemic optic neuropathy? J Neuroophthalmol 2012; 32: 51-53

22 Newman NJ, Scherer R, Langenberg P, Kelman S, Feldon S, Kaufman D et al. The fellow eye in NAION: report from the ischemic optic neuropathy decompression trial follow-up study. Am J Ophthalmol 2002; 134: 317-328.

23 Hayreh SS, Podhajsky PA, Zimmerman B. Ipsilateral recurrence of nonarteritic anterior ischemic optic neuropathy. Am J Ophthalmol 2001; 132: 734-742.

24 Pepple KL, Bhatti MT, Foroozan R. Not Again! Surv Ophthalmol 2011; 56: 86-93.

25 Ganssauge M, Wilhelm H, Bartz-Schmidt KU, Aisenbrey S. Non-arteritic anterior ischemic optic neuropathy (NA-AION) after intravitreal injection of bevacizumab (AvastinA (R)) for treatment of angoid streaks in pseudoxanthoma elasticum. Graefes Arch Clin Exp Ophthalmol 2009; 247: 1707-1710.

26 Huang JY, Ozaki H, Hayashi H, Uchio E. Anterior ischemic optic neuropathy following intravitreal bevacizumab. Jpn J Ophthalmol 2010; 54: 252-254.

27 Hosseini H, Razeghinejad MR. Anterior ischemic optic neuropathy after intravitreal injection of bevacizumab. J Neuroophthalmol 2009; 29: 160-161.

28 Falkenstein IA, Cheng L, Freeman WR. Changes of intraocular pressure after intravitreal injection of bevacizumab (avastin). Retin-J Retin Vitr Dis 2007; 27: 1044-1047.

29 Hollands H, Wong J, Bruen R, Campbell RJ, Sharma S, Gale J. Short-term intraocular pressure changes after intravitreal injection of bevacizumab. Can J Ophthalmol 2007; 42: 807-811.

30 Hayreh SS, Zimmerman MB. Incipient nonarteritic anterior ischemic optic neuroplathy. Ophthalmology 2007; 114: $1763-1772$. 